\title{
Refractive Index Control with Using C-S-Au Compound by Plasma CVD and Sputtering
}

\author{
Masaki Matushita, Md. Abul Kashem and Shinzo Morita \\ Department of Electronics, Nagoya University \\ Furo-cho, Chikusa-ku, Nagoya 464-8603, Japan \\ matusita@tcp-ip.or.jp
}

\begin{abstract}
$\mathrm{C}-\mathrm{S}$ and C-S-Au compound were formed by $\mathrm{RF}$ plasma $\mathrm{CVD}$ with using methane, $\mathrm{SF}_{6}$ and $\mathrm{Ar}$ mixture gas at a pressure of $0.1 \mathrm{Torr}$, a discharge frequency of $13.56 \mathrm{MHz}$ and a discharge power of 100 Watt, and a discharge duration of $30 \mathrm{~min}$, but C-S-Au compound was formed using gold plate set on the upper graphite discharge electrode instead of graphite plate. At the constant gas flow rate of 10 SCCM for methane and Ar, and the varying $\mathrm{SF}_{6}$ flow rate from 2 to $25 \mathrm{SCCM}$, the refractive indexes were changed from 1.7 to 2.4 and from 2.0 to 3.7 for C-S and C-S-Au compound, respectively.
\end{abstract}

Keywords: C-S-Au compound, C-S compound, RF plasma CVD, Refractive Index

\section{Introduction}

Recently various carbonaceous films are developing, which are interested in those unique characteristics. Amorphous carbon film with ultraviolet rays shielding property is applied to the coating of pet bottle for beer and other chemicals, which has not the ultraviolet rays resistance [1]. Low dielectric constant fluorinated carbonaceous film is important as insulator to decrease the time lag of information transfer of conductor in the integrated circuit. More recently, conducting amorphous carbon film is interested as the cathode emitter for the flat panel display [2].

Amorphous carbonaceous films are aimed to use as dielectrics in a photonic crystal [3], because different refractive index materials can be formed easily in the same reactor. Therefore photonic crystal can be fabricated by multiplying the two different refractive index materials and the carbonaceous films can be processed easily by a simple etching process.

Metal containing carbonaceous film is supposed to be a high refractive index material because a large atomic number material (high Z) usually shows large electronic polarization. Actually C-Au compound film showed a large refractive index [4].

In the previous works [5], C-S compound were successfully formed by RF plasma $\mathrm{CVD}$ with using methane, $\mathrm{SF}_{6}$ and $\mathrm{Ar}$ mixture gas. Therefore $\mathrm{C}-\mathrm{A}-\mathrm{Au}$ compound was formed with using the same gas composition and gold plate set on the upper discharge electrode and the refractive indexes were measured for both of C-S and C-S-Au compound in this work.

\section{Experimental}

The capacitively coupled RF plasma CVD reactor was used for the CDV at a discharge frequency of $13.56 \mathrm{MHz}$ [6]. The upper electrode of graphite plate with numerous small holes for the reaction gas supply was connected to the RF power source and the lower electrode was grounded for the CVD of C-S composite films. To form gold containing film, gold $(\mathrm{Au})$ plate was set on the upper electrode to be sputtered during the plasma CVD process. Methane, $\mathrm{SF}_{6}$ and $\mathrm{Ar} / \mathrm{H}_{2}$ mixture gas was used for the plasma 
CVD. The condition for the plasma CVD was constant at a pressure of 0.1 Torr, a RF discharge power of $100 \mathrm{~W}$, and discharge duration of $30 \mathrm{~min}$.

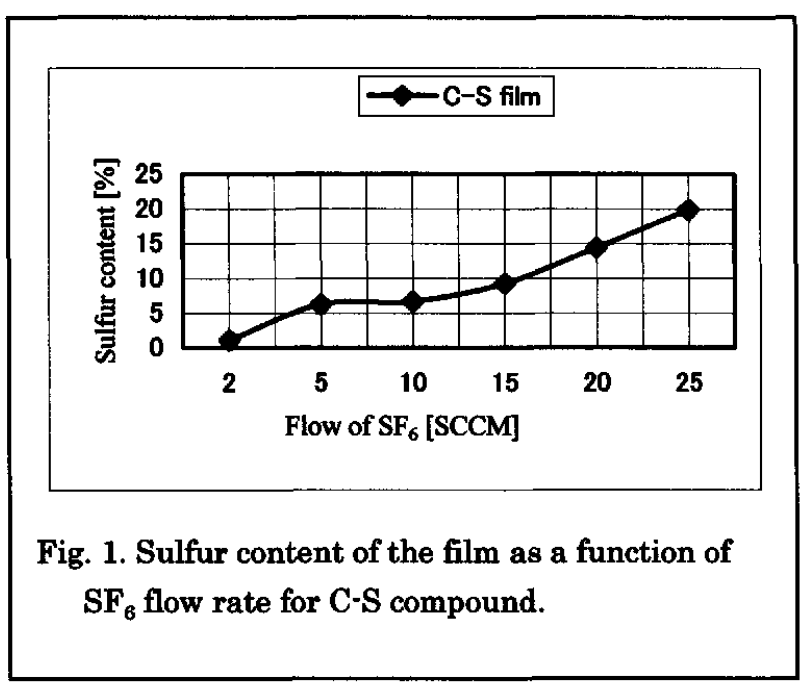

\section{Results and Discussions}

For the mixture gas of methane, $\mathrm{SF}_{6}$ and $\mathrm{Ar}, \mathrm{C}-\mathrm{S}$ compound film was formed by the plasma CVD and the sulfur content was measured by ESCA as shown in Fig. 1. When the methane and $\mathrm{Ar}$ flow rate were kept to be constant at $10 \mathrm{SCCM}$, and the flow rate of $\mathrm{SF}_{6}$ flow rate was varied from 2 to $25 \mathrm{SCCM}$, the sulfur content was increased from 2 to 20 atomic \%, but other atomic content of fluorine and oxygen were smaller than a few atomic \%, where the ESCA measurements were performed for the bulk after the surface layer was sputtered by Ar plasma before the measurement.

In our previous works with using same mixture gas at 2 SCCM for methane, 20 SCCM for Ar and 1 to $50 \mathrm{SCCM}$ for $\mathrm{SF}_{6}$, the sulfur content showed the peak of 30 atomic \% at $10 \mathrm{SCCM}$ of $\mathrm{SF}_{6}$. At the higher flow rate of $\mathrm{SF}_{6}$, fluorine atom content was increased and saturated to 40 atomic $\%$. Therefore the chemistry of $\mathrm{SF}_{6}$ decomposition was understood as that $\mathrm{SF}_{6}$ would be decomposed by detaching $\mathrm{F}$ atom by reacting with atomic $\mathrm{H}$ dissociated from the methane in the plasma. At the higher flow rate of $\mathrm{SF}_{6}, \mathrm{H}$ atom number was too small compared to $F$ atom in the gas phase, whereas $\mathrm{SF}_{6}$ could not be decomposed to the atom completely [5].

For the increased methane flow rate in this work, the monotonic increase of sulfur content means that $\mathrm{SF}_{6}$ was completely decomposed.

In order to increase sulfur content in the compound film, the methane, $\mathrm{SF}_{6}$ and $\mathrm{H}_{2}$ mixture gas was used because the hydrogen content in the gas phase can be controlled easily [7]. However, the content of sulfur in the film was limited to be $10-20$ atomic $\%$ for the wide flow rate variation of $\mathrm{SF}_{6}$. This fact means volatile sulfide molecules like as $\mathrm{H}_{2} \mathrm{~S}$ were formed.

$\mathrm{Au}$ containing $\mathrm{C}-\mathrm{S}$ compound films were formed with using the same gas mixture at the same gas flow rate but only changing the upper electrode material. The reason why the gold was mixed into the C-S composite film is explained by sputtering phenomena of gold plate on the upper electrode. The parallel discharge electrodes in the reactor have same diameter but the lower electrode was grounded with the reactor vessel. Therefore the total electrode area for the discharge of the grounded electrode is larger than the upper electrode connected to RF power source. During the discharge, the discharge current from both electrodes is same, but the discharge current density different. Namely the upper electrode discharge current density is larger than that of the lower grounded electrode. Whereas the upper electrode works as a cathode and negatively biased. The biased cathode usually bombarded continuously by positive ions. If the bombarding ion energy is high enough to sputtering the electrode materials, the upper electrode will be sputtered during the plasma CVD. In this experiment, the operation pressure was 0.1 Torr. This pressure is not so small compared to usual sputtering experiments. We must consider another 
Table 1. Atomic composition of the C-S-Au film.

( $\mathrm{CH}_{4}$ : 10 SCCM, Ar: 10 SCCM, Pressure : 0.1 Torr.)

\begin{tabular}{|c|c|c|c|c|c|}
\hline Flow of SF $_{6}$ (SCCM) & $\mathrm{C}(\%)$ & $\mathrm{S}(\%)$ & $\begin{array}{c}\mathrm{O} \\
(\%)\end{array}$ & $\begin{array}{c}\mathrm{F} \\
(\%)\end{array}$ & $\begin{array}{c}\mathrm{Au} \\
(\%)\end{array}$ \\
\hline 2 & 91.6 & 2.4 & 4.9 & 0.3 & 0.8 \\
\hline 5 & 90.0 & 4.6 & 3.7 & 1.1 & 0.5 \\
\hline 10 & 91.6 & 6.5 & 0.9 & 0.7 & 0.3 \\
\hline 15 & 87.9 & 7.6 & 1.3 & 3.1 & 0.05 \\
\hline 20 & 83.2 & 14.3 & 0.6 & 1.5 & 0.4 \\
\hline 25 & 79.2 & 18.7 & 1.2 & 0.8 & 0.1 \\
\hline
\end{tabular}

to 2.4 with increasing the $\mathrm{SF}_{6}$ flow rate from 2 to 25 SCCM. For C-S-Au compound, the refractive indexes were increased also monotonically from 2.0 to 3.7 for the same gas flow rate change of $\mathrm{SF}_{6}$.

Au containing C-F compound showed a large refractive index of assistance for the Au elimination from the upper electrode. It may be chemical assistance of $F$ atoms for the etching. However the details were not elucidated in this work.

The atomic compositions for C-S-Au compound were measured as shown in Table 1 in the bulk. With increasing $\mathrm{SF}_{6}$ flow rate, the sulfur content was increased monotonically, too. However the Au content was very small and about 0.05 to 0.8 atomic \% but did not showed the $\mathrm{SF}_{6}$ flow rate dependence. $\mathrm{F}$ and $\mathrm{O}$ atom were not neglected but very small compared to $C$ and $\mathrm{S}$ atom content. The origin of oxygen in the bulk will be referred to leaked air into the reactor, because surface layer was sputtered.

After the deposition, we observed some material on the Au plate at the upper electrode. The deposited material was sometimes wiped away by paper but could not be dissolved in the organic solvent. The deposited material was expected to be black carbonaceous powder. The details of powder formation are not known presently. However it is expected that the powder formation interrupt the sputtering of gold. The small $\mathrm{Au}$ content in the C-S-Au compound will be referred to the above effect.

For the deposited C-S and C-S-Au composite film, the refractive indexes were measured by an elipsometer as shown in Fig. 2. For the C-S compound, the refractive index was increased monotonically from 1.7 about 2.9. Whereas it will be concluded that the increase of refractive index of C-S and C$\mathrm{S}-\mathrm{Au}$ compound compared to amorphous carbon film with a refractive index of 1.5 to 1.6 will be referred to sulfur and gold atom.

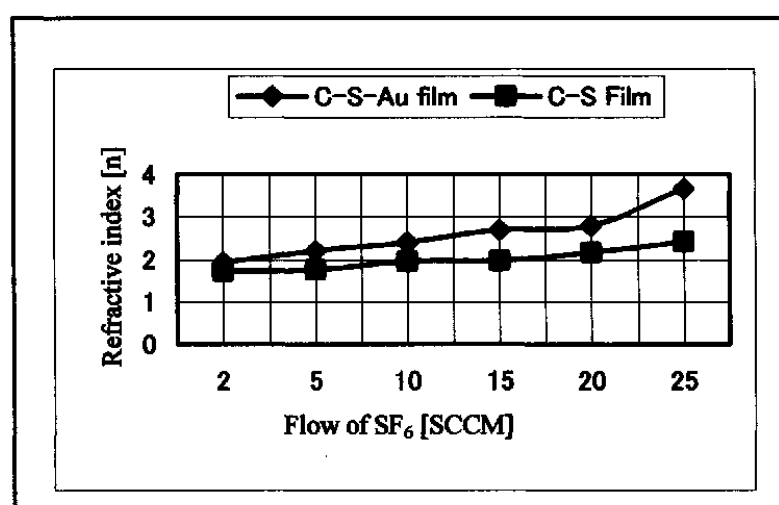

Fig. 2. Refractive indexes of $\mathrm{C}-\mathrm{S}$ and $\mathrm{C}-\mathrm{S}-\mathrm{Au}$ compound film as a function of $\mathrm{SF}_{6}$ flow rate .

\section{Conclusion}

We successfully fabricated C-S and C$\mathrm{S}-\mathrm{Au}$ compound film on the grounded electrode with using a mixture gas of methane, $\mathrm{Ar}$ and $\mathrm{SF}_{6}$ and $\mathrm{Au}$ plate on the upper electrode connected to the RF power source. The refractive indexes of C-S and C$\mathrm{S}-\mathrm{Au}$ composite film are from 1.7 to 2.4 and from 2.0 to 3.7 for the SF6 flow rate from 2 to 25 SCCM, respectively.

The C-S-Au compound film is an excellent candidate for a photonic crystal material because carbonaceous film and C-S- 
Au composite film are deposited in the same reactor only by changing the gas composition and the upper electrode.

\section{References}

[1] K. Nagashima, H. Shima, Japan Patent No. 2788412, Aug. 11(1994).

[2] G. A. J. Amaratunga and S. R. P. Silva, Appl. Phys. Lett., 68 (1996)2529.

[3] J. D. Joannopoulos, R. D. Meade, and J.

N. Winn; "Photonic Crystals", Princeton Univ. Press (1995).
[4] L. Marutinu, Solar Energy Materials, 15 (1987) 21.

[5] M. Matsushita, Md. Zarid. Bin Harum, Md. Abul Kashem, and S. Morita; J. Photoplolym. Sci. and Tech., 12 (1) (1999)11-14.

[6] M. matsushita, and S. Morita; J. Photoplolym. Sci. and Tech., 11 (2) (1998)257-262.

[7] Md. Abul kashem, M. Matsushita and S. Morita; J. Photoplolym. Sci. and Tech., 13 (1) (2000) 47-50. 